\title{
Optimising perioperative care for hip and knee arthroplasty in South Africa: a Delphi consensus study
}

\author{
U. Plenge ${ }^{1 *}$ D, M. B. Nortje ${ }^{2}$, L. C. Marais ${ }^{3}$, J. D. Jordaan ${ }^{4}$, R. Parker ${ }^{1}$, N. van der Westhuizen ${ }^{5}$, J. F. van der Merwe ${ }^{6}$, \\ J. Marais ${ }^{7}$, W. V. September ${ }^{7}$, G. L. Davies ${ }^{8}$, T. Pretorius ${ }^{8}$, C. Solomon ${ }^{9}$, P. Ryan ${ }^{10}$, A. M. Torborg ${ }^{11}$, Z. Farina ${ }^{12}$, \\ R. Smit ${ }^{13}$, C. Cairns ${ }^{14}$, H. Shanahan ${ }^{15}$, S. Sombili ${ }^{16}$, A. Mazibuko ${ }^{17}$, H. R. Hobbs ${ }^{2}$, O. S. Porrill ${ }^{1}$, N. E. Timothy ${ }^{18}$, \\ R. E. Siebritz ${ }^{18}$, C. van der Westhuizen ${ }^{19}$, A. J. Troskie ${ }^{20}$, C. A. Blake ${ }^{20}$, L. A. Gray ${ }^{21}$, T. W. Munting ${ }^{22}$, \\ H. K. S. Steinhaus ${ }^{23}$, P. Rowe ${ }^{24}$, J. G. van der Walt ${ }^{25}$, R. Isaacs Noordien ${ }^{26}$, A. Theron ${ }^{27}$ and B. M. Biccard ${ }^{1}$
}

\begin{abstract}
Background: A structured approach to perioperative patient management based on an enhanced recovery pathway protocol facilitates early recovery and reduces morbidity in high income countries. However, in low- and middle-income countries (LMICS), the feasibility of implementing enhanced recovery pathways and its influence on patient outcomes is scarcely investigated. To inform similar practice in LMICs for total hip and knee arthroplasty, it is necessary to identify potential factors for inclusion in such a programme, appropriate for LMICs.

Methods: Applying a Delphi method, 33 stakeholders (13 arthroplasty surgeons, 12 anaesthetists and 8 physiotherapists) from 10 state hospitals representing 4 South African provinces identified and prioritised i) risk factors associated with poor outcomes, ii) perioperative interventions to improve outcomes and iii) patient and clinical outcomes necessary to benchmark practice for patients scheduled for primary elective unilateral total hip and knee arthroplasty.
\end{abstract}

Results: Thirty of the thirty-three stakeholders completed the 3 months Delphi study. The first round yielded i) 36 suggestions to preoperative risk factors, ii) 14 (preoperative), 18 (intraoperative) and 23 (postoperative) suggestions to best practices for perioperative interventions to improve outcomes and iii) 25 suggestions to important postsurgical outcomes. These items were prioritised by the group in the consecutive rounds and consensus was reached for the top ten priorities for each category.

Conclusion: The consensus derived risk factors, perioperative interventions and important outcomes will inform the development of a structured, perioperative multidisciplinary enhanced patient care protocol for total hip and knee arthroplasty. It is anticipated that this study will provide the construct necessary for developing pragmatic enhanced care pathways aimed at improving patient outcomes after arthroplasty in LMICs.

Keywords: Delphi study, Enhanced recovery pathways, Low middle income countries, Total hip and knee arthroplasty, Total hip and knee replacement, Patient outcomes

\footnotetext{
* Correspondence: ullaplenge@gmail.com

${ }^{1}$ Department of Anaesthesia and Perioperative Medicine, Groote Schuur Hospital and Faculty of Health Sciences, University of Cape Town, Cape Town, South Africa

Full list of author information is available at the end of the article
}

(c) The Author(s). 2018 Open Access This article is distributed under the terms of the Creative Commons Attribution 4.0 International License (http://creativecommons.org/licenses/by/4.0/), which permits unrestricted use, distribution, and reproduction in any medium, provided you give appropriate credit to the original author(s) and the source, provide a link to the Creative Commons license, and indicate if changes were made. The Creative Commons Public Domain Dedication waiver (http://creativecommons.org/publicdomain/zero/1.0/) applies to the data made available in this article, unless otherwise stated. 


\section{Background}

In the past 20 years, enhanced recovery pathways (ERPs) have become increasingly integrated into most surgical fields as standard care in high income countries, as is exemplified by national priority programs [1-3], and the widespread acceptance of the Enhanced Recovery After Surgery (ERAS) society network [4]. ERPs represent a fundamental shift towards a patient-centred, multidisciplinarydriven continuity of care that aim to attenuate surgical stress and expedite recovery [5]. Studies on total joint arthroplasty (TJA) for both hips and knees have shown that implementation of an evidence-based, structured approach to patient care decreases postoperative morbidity and consequently length of stay without increasing readmission rate [6-8].

However, in low- and middle-income countries (LMICs) , the value of implementing ERPs is yet to be explored. This may be because: i) the perception that current hospital resources may make it difficult to develop and implement structured and sustainable protocols to enhance postoperative recovery, and ii) short and long-term data collection on the quality of the work provided is scarce, inhibiting the ability to benchmark clinical results and improve the service provided to patients. Despite these challenges, a healthcare system in a middle-income country such as South Africa may benefit from the implementation of ERPs through reduced postoperative morbidity and the associated cost reductions, as has been demonstrated in high-income countries (HICs) [9].

While the goals of implementing ERPs can be expected to be independent of a country's economic status, we believe the differences in patient demographics, healthcare infrastructure and healthcare resources between HICs and LMICs warrants a LMIC derived programme of enhanced care to facilitate practice change and improve patient outcomes in these settings. The aim of our study was therefore to establish multidisciplinary consensus on; i) preoperative risk factors associated with poor outcomes, ii) perioperative interventions considered necessary to improve outcomes, and iii) important postsurgical patient and clinical outcomes. This study was conducted in South Africa, which represents an upper-middle-income country, as defined by the World Bank [10]. However, as this work was conducted in the public healthcare sector, and South Africa has one of the world's highest levels of inequality [11], it is likely that this work reflects the state funded healthcare system of a LMIC, as opposed to highmiddle-income countries. This assumption is supported by the South African public healthcare service data from the African Surgical Outcomes Study, where the median number of specialists per 100,000 population was 0.9 (IQR 0.2-1.9) (unpublished data) [12], which is well below the recommended $20-40$ specialists per 100,000 population [13].

\section{Methods}

We conducted a Delphi survey with experts from different fields involved in the care of arthroplasty surgical patients in South Africa. The Delphi study is an accepted method for achieving convergence of opinions concerning knowledge solicited from experts within specific fields, and has been adopted for priority-setting in medicine [14]. The technique is an iterative process which allows the participant to refine his or her prioritization of items, in an anonymous manner, based on the group's work from round to round and with controlled feedback of opinions [15].

\section{Participant recruitment}

Participants were recruited from all the hospitals which we knew had a history of performing elective TJAs. This approach was necessary, as currently there is no national arthroplasty database of public hospitals performing TJAs in South Africa. We invited orthopaedic arthroplasty surgeons, anaesthetists and physiotherapists from 18 regional and central hospitals in the public sector covering seven of the nine provinces in South Africa. They were contacted by email and asked to participate in four sequential studies aimed at improving perioperative care for patients scheduled for primary elective unilateral hip and knee TJA in South Africa. The Delphi study is the first of these four studies. For a hospital to participate we required participation of both the Anaesthesia and the Orthopaedic Departments in the project. With the use of telephone calls, face-to-face meetings and further email correspondence, 33 experts in the perioperative management of arthroplasty patients from 10 hospitals representing four provinces accepted the invitation to participate in these four studies. Reasons for exclusion from the study where i) not confirming their participation (5) or ii) declining to participate due to lack of interest or lack of resources to participate in this and future studies (3). Prior to commencement of the Delphi study, the participants were given detailed information of the Delphi process and how consensus would be defined.

\section{The Delphi process}

This Delphi survey was conducted over 3 months from December 2016 to March 2017. In the first round participants submitted suggestions for; i) risk factors associated with poor outcome, ii) best practices for preoperative, intraoperative and postoperative interventions to improve postoperative outcomes and iii) important patient and clinical outcomes to benchmark care, deemed relevant in the South African context for patients scheduled for primary elective unilateral hip and knee TJA. Participants were encouraged to elaborate on how to quantify these components and provide 
supporting references. UP and BMB grouped the responses in each category into statements. The category statements and supporting references were shared with all participants. In the second Delphi round, the participants were asked to rank the top-ten statements in each category, and where possible, add further comments or relevant references. Based on participants' responses, statements that overlapped were grouped together prior to the third Delphi round. In the third round the participants were presented with their individual as well as the overall group ranking of the prioritised statements within each category. They were asked to re-evaluate their previous round's ranking, considering the group ranking and where possible when their rankings differed greatly from that of the group, to add further comments or references supporting their decision. In the fourth and final round, participants were given an opportunity to present any strong disagreement with the priority rankings from the third Delphi round with a Skype teleconference. Non-participation in the fourth round indicated agreement with the proposed Delphi priorities from the third round. Following the teleconference, the consensus of the group was taken as final. UP and BMB were neutral in the prioritization of statements throughout the study.

\section{Statistical analysis}

The rank order of the research priorities for each round was established using a reverse scoring system i.e. a respondent's rank of 1 received 10 points, down to a rank of 10 , which received 1 point. The scores of the respondents were combined for each round to develop the research priority rank order.

\section{Results}

\section{Participants and response rate}

The recruited participants included 13 arthroplasty surgeons, 12 anaesthetists and 8 physiotherapists involved in hip and knee arthroplasty. Response rate in the first round was 97\% (32/33), 91\% (30/33) in the second round and 91\% (30/33) in the third round. In the fourth round, all 33 participants accepted the ranking of the prior third Delphi round. However, three participants contributed in the fourth round to a refinement of two of the Delphi statements. The first was an amalgamation of "peripheral nerve blocks" with "multimodal opioidsparing analgesia regimen" in the postoperative intervention category, which changed the overall ranking in this category. This change clarified that non-opioid analgesic regimens can include regional anaesthesia. The second change was to define "long term survival" in the outcome category as "1-year mortality", to ensure an objective outcome variable.

\section{Preoperative risk factors}

Two hundred forty-seven suggestions were submitted for round 1 for preoperative risk factors believed to be associated with poor outcomes in patients scheduled for primary elective unilateral hip and knee TJA. The suggestions were categorised into 36 broad statements for round 2 which were refined to 28 statements for round 3 . The ten prioritised risk factors identified after the second round did not change in the subsequent rounds (Table 1).

\section{Preoperative interventions}

Round 1 yielded 166 suggestions of preoperative interventions judged to be important to improve outcomes following primary elective unilateral hip and knee TJA. These were amalgamated into 14 statements in round 2 and further refined to 11 different statements for round 3 . The ten priorities identified after the second round did not change in subsequent rounds (Table 2).

\section{Intraoperative interventions}

One hundred forty-four suggestions for intraoperative interventions believed to improve postoperative outcomes following primary elective unilateral hip and knee TJA were submitted in round 1 . These were amalgamated into 18 statements for the second round and further refined to 11 statements for round 3. The ten priorities identified by the second round, did not change in the fourth round (Table 3).

\section{Postoperative interventions}

The first Delphi round yielded 181 suggestions of important postoperative interventions to possibly improve outcomes following primary elective unilateral hip and knee TJA. These were amalgamated into 23 statements for the second Delphi round and further refined to 17

Table 1 The ten prioritised preoperative risk factors considered most important determinants of poor outcomes in patients scheduled for primary elective unilateral hip and knee total joint arthroplasty in South Africa

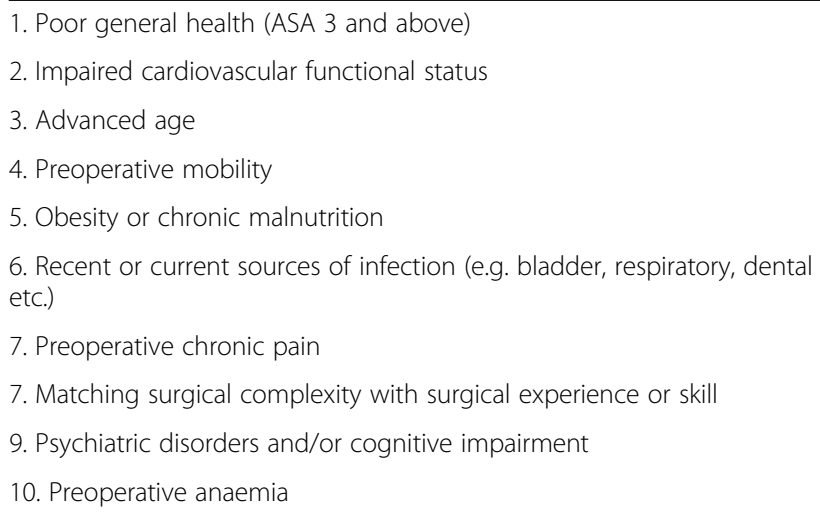

ASA American Society of Anesthesiologists 
Table 2 The ten prioritised preoperative interventions considered most important determinants to improve outcomes following primary elective unilateral hip and knee total joint arthroplasty in South Africa

1. A patient optimisation clinic
2. Multidisciplinary planning
3. Patient education
4. Infection prevention
5. Establishing high-volume units
6. Smoking cessation
7. Optimisation of preoperative analgesia regimen
8. Minimise preoperative fasting
9. Establish a patient blood management programme
10. Alcohol cessation

statements for the third Delphi round. The final ten priorities were agreed upon in the fourth round of the Delphi process, following amalgamation of "peripheral nerve blocks" into "multimodal opioid-sparing analgesia regimen" (Table 4).

\section{Important patient and clinical outcomes}

One hundred sixty-four suggestions were made in the first Delphi round for important patient and clinical outcomes following primary elective unilateral hip and knee TJA. These were categorised into statements for the second Delphi round and further refined to 23 statements for the third Delphi round. The ten prioritised outcomes did not change after the second round (Table 5).

\section{Discussion}

This study reports a national consensus of the predictors of morbidity, perioperative interventions to improve surgical outcomes, and the clinical outcomes necessary to document perioperative success for patients scheduled

Table 3 The ten prioritised intraoperative interventions considered most important determinants to improve outcomes following primary elective unilateral hip and knee total joint arthroplasty in South Africa

1. Meticulous surgical technique
2. Infection prevention
3. Optimisation of prosthesis choice and placement
4. Multimodal opioid-sparing analgesia regimen
5. Monitoring and optimisation of haemodynamics
6. Central neuraxial anaesthesia
7. Establish a patient blood management programme
8. Temperature regulation
9. Glycaemic control
10. Deep vein thrombosis prophylaxis

Table 4 The ten prioritised postoperative interventions considered most important determinants to improve outcomes following primary elective unilateral hip and knee total joint arthroplasty in South Africa

1. Early mobilisation after surgery

2. Standardised orthopaedic nursing care

3. Multimodal opioid-sparing analgesia regimen

4. Active management of medical co-morbidities

5. DVT prophylaxis

6. A pain management team

7. Patient empowerment in his or her recovery

8. Patient controlled analgesia

9. Multidisciplinary ward rounds

10. Postoperative rehabilitation

for primary elective unilateral hip and knee TJA in South Africa. These findings provide the information necessary to develop a feasible enhanced care programme for South African arthroplasty patients.

The multidisciplinary involvement of regional and central hospitals performing TJAs across South Africa provides a realistic consensus of the factors needed for an enhanced care arthroplasty programme in the public service in South Africa. We believe that the "buy-in" by the participants was high, and this is important for successful organisational change [16]. Furthermore, consensus on the priorities was established early (within Delphi round 2) in four of the five categories, supporting the validity of the final consensus document [17].

However, this study also has limitations. Firstly, while expert consensus is the lowest level of evidence, it is an established method to facilitate clinical guidelines when the evidence is limited [18], particularly when study interventions and study results might not be transferable to settings with a different socio-economic and demographic structure. Furthermore, group consensus studies

Table 5 The ten prioritised patient and clinical outcomes considered most important following primary elective unilateral hip and knee total joint arthroplasty in South Africa
1. Patient reported outcome measures
2. Postoperative pain at rest and during movement
3. 1-year mortality
4. Early mobilisation
5. Prosthetic joint infection rate
6. Joint range of motion
7. Major adverse cardiac events
8. Hospital length of stay
9. Implant longevity
10. Cost of care 
can expedite the transformation of evidence-based knowledge gained in HICs into practical implementation in LMICs [19], which is why we believe this process is entirely appropriate for the public health service in South Africa, and may be applicable to other LMICs. In our study we have: i) identified feasible interventions which may improve patient outcomes in a resource limited environment, and ii) prioritized which interventions are preferable for implementation if resources do not allow for adoption of all suggested interventions in clinical practice. We believe this approach will allow all sites to focus their resources on developing a pragmatic multidisciplinary programme of enhanced care.

A second limitation is the possibility that we did not invite all sites which performs TJAs in South Africa to participate in the study, as the public health care sector currently does not have a national arthroplasty database. Nevertheless, we succeeded in enrolling both regional and central hospitals from different provinces, which ensured a broad representation of specialists involved in TJAs in South Africa. Finally, we did not include the full spectrum of stakeholders involved in the perioperative management of joint arthroplasty patients or patients themselves. However, we believe our consensus document does represent stakeholders who were not participants in this study, as patient relevant outcomes and parameters important to nursing care, physicians, nutritionists and geriatricians are included (Tables 1, 2, 3, 4 and 5).

Identification of modifiable and non-modifiable risk factors is essential to guide surgical decision making and prepare the patient optimally ensuring safe perioperative care [20]. This is important in a country such as South Africa, which has a medium Human Development Index $(\mathrm{HDI})^{1}$ suggesting a higher risk for perioperative mortality compared to countries with high HDI [21]. Hence, addressing the prioritised preoperative risk factors (Table 1) may improve patient outcomes [22]. Additionally, introducing a best practice protocol in the perioperative period (Tables 2, 3 and 4) aims to provide continuity of care with emphasis on less variability and better quality of service provided [23]. Finally, identifying and standardising procedure specific outcomes facilitates benchmarking, which is crucial to improve the quality of patient care [24]. Only recently have such multinational collaborative efforts been instituted for TJAs to guide future trials towards comparable outcomes [25]. Importantly, this international group of patient partners, orthopaedic surgeons, physical therapists, rheumatologists and methodologists successfully achieved consensus for six core outcome domains; i) pain, ii) function, iii) patient satisfaction, iv) revision, v) adverse events and vi) death, which are all represented in our consensus document (Table 5). While this similarity provides external validity to the work of our Delphi group, it also suggests that aspirations for best patient practice is independent of a country's income status. However, the novelty of our Delphi study remains with the prioritised preoperative risk factors and perioperative interventions, which we hope will facilitate a pragmatic approach to achieving these postoperative goals in our resource limited settings.

\section{Conclusion}

This national multidisciplinary consensus Delphi study has produced priorities for preoperative risk stratification, perioperative interventions, and outcome assessments necessary for benchmarking, from which a pragmatic enhanced care programme for primary elective unilateral hip and knee TJA in South Africa can be developed. It is anticipated that these priorities may either be applicable or encourage other LMICs to initiate a similar Delphi process. The next phase will involve an audit of current perioperative care addressing the prioritised statements, followed by implementation of the Delphi group's proposed interventions.

\section{Endnotes}

${ }^{1 *}$ Human Development Index (HDI) is an index based on i) life expectancy, ii) education and iii) per capita income indicators, which is used to determine whether a nation is a developed or a developing country.

\section{Abbreviations \\ ASA: American Society of Anesthesiologists; DVT: Deep vein thrombosis; ERAS: Enhanced recovery after surgery; ERPs: Enhanced recovery pathways; HDI: Human Development Index; HICs: High-income countries; LMICs: Low- and middle-income countries; PBM: Patient blood management; TJA: Total joint arthroplasty}

\section{Availability of data and materials}

The datasets used and analysed during the current study are available from the corresponding author on request.

\section{Authors' contributions}

UP and BMB were responsible for overall conception and design of the Delphi study; acquisition, analysis and interpretation of data; drafting the manuscript and critical revising of the work. MBN, LCM, JDJ, RP, NvdW, JFVdM, JM, WVS, GLD, TP, CS, PR, AMT, ZF, RS, CC, HS, SS, AM, HRH, OSP, NET, RES, CvdW, AJT, CAB, LAG, TWM, HKSS, PR, JGvdW, RIN and AT made substantial contributions to acquisition, analysis and interpretation of data and critical revising of the work. All authors approved the final version and agreed to be accountable for all aspects, accuracy and integrity of the work.

\section{Ethics approval and consent to participate}

The study was approved by the University of Cape Town, Faculty of Health Sciences Human Research Committee, South Africa; HREC REF: 807/2016. Written consent was obtained as participants consented to take part in the study by replying to each of the Delphi cycles via emails and their responses were stored in a password protected electronic format.

Competing interests

The authors declare that they have no competing interests. 


\section{Publisher's Note}

Springer Nature remains neutral with regard to jurisdictional claims in published maps and institutional affiliations.

\section{Author details}

'Department of Anaesthesia and Perioperative Medicine, Groote Schuur Hospital and Faculty of Health Sciences, University of Cape Town, Cape Town, South Africa. ${ }^{2}$ Department of Orthopaedic Surgery, Groote Schuur Hospital and Faculty of Health Sciences, University of Cape Town, Cape Town, South Africa. ${ }^{3}$ Department of Orthopaedic surgery, School of Clinical Medicine, University of KwaZulu-Natal, Pietermaritzburg, South Africa. ${ }^{4}$ Department of Orthopaedic Surgery, Tygerberg Medical School, University of Stellenbosch, Cape Town, South Africa. ${ }^{5}$ Department Anaesthesia, University of the Free State, Bloemfontein, South Africa. ${ }^{6}$ Department of Orthopaedic surgery, University of the Free State, Bloemfontein, South Africa. 7 Department of Physiotherapy, Paarl Provincial Hospital, Paarl, South Africa. ${ }^{8}$ Department of Anaesthesia, Paarl Provincial Hospital, Paarl, South Africa. ${ }^{9}$ Department of Orthopaedics, Paarl Provincial Hospital, Paarl, South Africa.

${ }^{10}$ Arthroplasty and Sports Medicine unit, Department of Orthopaedics, Inkosi Albert Luthuli Central Hospital, University of KwaZulu-Natal, Durban, South Africa. ${ }^{11}$ Department of Anaesthesia, Inkosi Albert Luthuli Central Hospital, University of KwaZulu-Natal, Durban, South Africa. ${ }^{12}$ Department of Anaesthesia, Critical Care and Pain Management, Grey's Hospital, Pietermaritzburg, South Africa. ${ }^{13}$ Department of Orthopaedic surgery, Grey's Hospital, Pietermaritzburg, South Africa. ${ }^{14}$ Greys Pain clinic, Department of Anaesthesia, Grey's Hospital, Pietermaritzburg, South Africa. ${ }^{15}$ Department of Physiotherapy, Grey's Hospital, Pietermaritzburg, South Africa. ${ }^{16}$ Department of Orthopaedic surgery, Steve Biko Academic Hospital, University of Pretoria, Pretoria, South Africa. ${ }^{17}$ Department of Anaesthesia, Steve Biko Academic Hospital, University of Pretoria, Pretoria, South Africa. ${ }^{18}$ Department of Physiotherapy, Groote Schuur Hospital and Faculty of Health Sciences, University of Cape Town, Cape Town, South Africa. ${ }^{19}$ Department of Anaesthesia, Worcester Hospital, Worcester, South Africa. ${ }^{20}$ Department of Orthopaedic Surgery, Worcester Hospital, Worcester, South Africa. ${ }^{21}$ Department of Physiotherapy, New Somerset Hospital, Cape Town, South Africa. ${ }^{22}$ Department of Orthopaedics, New Somerset Hospital and Christiaan Barnard Memorial Hospital, Cape Town, South Africa. ${ }^{23}$ Department of Anaesthesia, New Somerset Hospital, Cape Town, South Africa. ${ }^{24}$ Department of Orthopaedic surgery, Victoria Hospital, Cape Town, South Africa. ${ }^{25}$ Department of Anaesthesia, Victoria Hospital, Cape Town, South Africa. ${ }^{26}$ Department of Physiotherapy, Victoria Hospital, Cape Town, South Africa.

${ }^{27}$ Department of Anaesthesiology and Critical Care, Tygerberg Academic Hospital, University of Stellenbosch, Cape Town, South Africa.

Received: 15 December 2017 Accepted: 26 April 2018

Published online: 09 May 2018

\section{References}

1. Alfonsi P, Slim K, Chauvin M, Mariani P, Faucheron JL, Fletcher D. French guidelines for enhanced recovery after elective colorectal surgery. J Visc Surg. 2014;151(1):65-79.

2. Simpson JC, Moonesinghe SR, Grocott MP, Kuper M, McMeeking A, Oliver CM, Galsworthy MJ, Mythen MG, National Enhanced Recovery Partnership Advisory B. Enhanced recovery from surgery in the UK: an audit of the enhanced recovery partnership programme 2009-2012. Br J Anaesth. 2015; 115(4):560-8.

3. Scott MJ, McEvoy MD, Gordon DB, Grant SA, Thacker JKM, Wu CL, Gan TJ, Mythen MG, Shaw AD, Miller TE. American Society for Enhanced Recovery (ASER) and Perioperative Quality Initiative (POQI) joint consensus statement on optimal analgesia within an enhanced recovery pathway for colorectal surgery: part 2-from PACU to the transition home. Perioper Med (Lond). 2017:6:7.

4. The ERAS Society. http://www.erassociety.org. Assessed on $20^{\text {th }}$ March 2018

5. Kehlet H. Multimodal approach to control postoperative pathophysiology and rehabilitation. Br J Anaesth. 1997;78(5):606-17.

6. Scott NB, McDonald D, Campbell J, Smith RD, Carey AK, Johnston IG, James KR, Breusch SJ. The use of enhanced recovery after surgery (ERAS) principles in Scottish orthopaedic units-an implementation and follow-up at 1 year, 2010-2011: a report from the musculoskeletal audit, Scotland. Arch Orthop Trauma Surg. 2013;133(1):117-24.

7. Kehlet H. Fast-track hip and knee arthroplasty. Lancet (London, England). 2013;381(9878):1600-2.
8. Zhu S, Qian W, Jiang C, Ye C, Chen X. Enhanced recovery after surgery for hip and knee arthroplasty: a systematic review and meta-analysis. Postgrad Med J. 2017;93:736.

9. Kash BA, Zhang Y, Cline KM, Menser T, Miller TR. The perioperative surgical home (PSH): a comprehensive review of US and non-US studies shows predominantly positive quality and cost outcomes. Milbank Q. 2014;92(4): 796-821.

10. The World Bank data on upper-middle-income countries. https://data.worldbank. org/income-level/upper-middle-income. Assessed on 20 th March 2018.

11. United Nations Human Development Report. http://hdr.undp.org/en/ composite/IHDI. Assessed on 20 $0^{\text {th }}$ March 2018.

12. Biccard BM, Madiba TE, Kluyts HL, Munlemvo DM, Madzimbamuto FD, Basenero A, Gordon CS, Youssouf C, Rakotoarison SR, Gobin V, et al. Perioperative patient outcomes in the African surgical outcomes study: a 7day prospective observational cohort study. Lancet (London, England). 2018; 391:1589.

13. Meara JG, Leather AJ, Hagander L, Alkire BC, Alonso N, Ameh EA, Bickler SW, Conteh L, Dare AJ, Davies J, et al. Global surgery 2030: evidence and solutions for achieving health, welfare, and economic development. Lancet (London, England). 2015;386(9993):569-624.

14. The James Lind Alliance. http://www.jla.nihr.ac.uk. Assessed on $20^{\text {th }}$ March 2018.

15. Hsu C-C, Sandford BA. The Delphi technique: making sense of consensus. Pract Assess Res Eval. 2007;12(10):1-8.

16. Campbell RJ. Change management in health care. Health Care Manag (Frederick). 2008;27(1):23-39.

17. Boulkedid R, Abdoul H, Loustau M, Sibony O, Alberti C. Using and reporting the Delphi method for selecting healthcare quality indicators: a systematic review. PLoS One. 2011;6(6):e20476.

18. Murphy MK, Black NA, Lamping DL, McKee CM, Sanderson CF, Askham J, Marteau T: Consensus development methods, and their use in clinical guideline development. Health Technol Assess 1998, 2(3):i-iv, 1-88.

19. Minas H, Jorm AF. Where there is no evidence: use of expert consensus methods to fill the evidence gap in low-income countries and cultural minorities. Int J Ment Health Syst. 2010;4:33.

20. Pearse RM, Holt PJ, Grocott MP. Managing perioperative risk in patients undergoing elective non-cardiac surgery. BMJ. 2011;343:d5759.

21. Bainbridge D, Martin J, Arango M, Cheng D, Evidence-based Peri-operative Clinical Outcomes Research G. Perioperative and anaesthetic-related mortality in developed and developing countries: a systematic review and meta-analysis. Lancet. 2012;380(9847):1075-81.

22. Levett DZH, Edwards M, Grocott M, Mythen M. Preparing the patient for surgery to improve outcomes. Best Pract Res Clin Anaesthesiol. 2016;30(2):145-57.

23. Garson L, Schwarzkopf R, Vakharia S, Alexander B, Stead S, Cannesson M, Kain Z. Implementation of a total joint replacement-focused perioperative surgical home: a management case report. Anesth Analg. 2014;118(5):1081-9.

24. Myles PS, Grocott MP, Boney O, Moonesinghe SR, Group CO-S. Standardizing end points in perioperative trials: towards a core and extended outcome set. Br J Anaesth. 2016;116(5):586-9.

25. Singh JA, Dowsey MM, Dohm M, Goodman SM, Leong AL, Scholte Voshaar $\mathrm{MM}$, Choong PF. Achieving consensus on total joint replacement trial outcome reporting using the OMERACT filter: endorsement of the final core domain set for total hip and total knee replacement trials for endstage arthritis. J Rheumatol. 2017;44:1723.

\section{Ready to submit your research? Choose BMC and benefit from:}

- fast, convenient online submission

- thorough peer review by experienced researchers in your field

- rapid publication on acceptance

- support for research data, including large and complex data types

- gold Open Access which fosters wider collaboration and increased citations

- maximum visibility for your research: over $100 \mathrm{M}$ website views per year

At BMC, research is always in progress.

Learn more biomedcentral.com/submissions 